\title{
Capacity and Performance of MIMO systems for Wireless Communications
}

\author{
E. Ghayoula ${ }^{1,2}$, A. Bouallegue ${ }^{1}$, R. Ghayoula ${ }^{2, *}$ and J-Y.Chouinard ${ }^{2}$ \\ ${ }^{I}$ Sys 'Com Laboratory, National Engineering School of Tunis, ENIT, Tunisia Tunis EL Manar University, Tunis, Tunisia \\ ${ }^{2}$ LRTS Laboratory, Department of Electrical and Computer Engineering, Laval University 1065 , Avenue de la Médecine, Québec
} (QC), Canada, G1V $0 A 6$

Received 14 March 2014; Accepted 1 August 2014

\begin{abstract}
This paper presents the capacity performance of multiple antennas for wireless communication systems. Multiple antennas structures can be classified into single-input multiple-outputs (SIMO), multiple-inputs single output (MISO), and multiple-inputs multiple-outputs (MIMO) systems. Assuming that the channel is unknown at receiver, capacity expressions are provided for each structure. Our results also show that increasing the number of transmitting and receiving antennas for a wireless MIMO channel does indeed improve the channel capacity that can be obtained.
\end{abstract}

Keywords: MIMO Systems; Capacity; SISO; SIMO; MISO; MIMO Channels; SNR.

\section{Introduction}

During the past decade, Wireless Communications and especially mobile communication systems have grown considerably. The radio communication systems must meet a growing number of users and no less demand for new services. New digital technologies are currently being studied to determine the fourth generation of mobile communication systems with the objectives to provide high spectral efficiency and a wide variety of services. In this context, communication systems MIMO (Multiple Input Multiple Output) based on the use of an antenna array at the transmitter and receiver are able to offer high-speed transmission with a minimum quality of service guarantee. The intense research on MIMO systems was inspired by seminal works by Telatar [11] and Foschini and Gans [12] that showed a dramatic linear increase in channel capacity with the number of antennas [13]. MIMO systems allow us to operate two distinct dimensions of a radio link; the first being the Diversity and the second being the Capacity. In this paper we will focus on the Capacity of MIMO channels because it is a performance measure for digital communication systems and it is the maximal transmission rate for which a reliable communication can be achieved.

This paper is organized as follows. Presentation of SISO, MISO, SIMO and MIMO Systems (Section II). Then we will present Capacity for all systems and we will concentrate to MIMO channels capacity in (Section III) and we will finish (Section IV) by a comparison between different multiantenna systems and the gain on capacity by increasing the number of antennas.

\section{MIMO Systems (SISO- MISO/SIMO-MIMO)}

\footnotetext{
*E-mail address: ridha_ghayoula_fst@yahoo.fr ISSN: 1791-2377 @ 2014 Kavala Institute of Technology. All rights reserved.
}

There are many formats of MIMO can be used from SISO, through SIMO and MISO to the full MIMO systems [6]. These are all able to provide significant improvements of performance, but generally at the cost of additional processing and the number of antennas used. Balances of performance against costs, size, processing available and the resulting battery life need to be made when choosing he correct option. Multiple-input and multiple-output, or MIMO is the use of multiple antennas at both the transmitter and receiver to improve communication performance. MIMO systems can be viewed as an extension of the socalled smart antennas, a popular technology using antenna arrays for improving wireless transmission dating back several decades [1]. Also it can offer huge advantages over single-antenna systems, both with regard to capacity and error performance. MIMO systems, which use multiple element antennas for signal transmission and reception, are expected to play a key role in improving the performance of future wireless communication systems [2], [3]. It has been shown that if the signal fading between pairs of transmit and receive antenna elements are independent and identically distributed (i.i.d.), the capacity of MIMO systems can increase linearly with the number of antennas [4]. Multipleinput multiple-output, or MIMO [5], is a radio communications technology or RF technology that is being mentioned and used in many new technologies these days. Wi-Fi, LTE; Long Term Evolution, and many other radio, wireless and RF technologies are using the new MIMO wireless technology to provide increased link capacity and spectral efficiency combined with improved link reliability using what were previously seen as interference paths. A MIMO system includes $\mathrm{N}$ transmission antennas and $\mathrm{M}$ reception antennas shown in (1), $\mathrm{x}$ is a vector with $\mathrm{N}$ symbols $[11,14]$ :

$\mathrm{x}=\left[\mathrm{x}_{1} \ldots \mathrm{x}_{\mathrm{t}} \ldots \mathrm{x}_{\mathrm{N}}\right]^{\mathrm{T}}$

$\mathrm{H}$ is the channel matrix of size $\mathrm{N} \times \mathrm{M}$. [6], [7], [14] 
$\mathrm{H}=\left[\begin{array}{ccc}\mathrm{h}_{11} & \cdots & \mathrm{h}_{1 \mathrm{~N}} \\ \vdots & \ddots & \vdots \\ \mathrm{h}_{\mathrm{M} 1} & \cdots & \mathrm{h}_{\mathrm{MN}}\end{array}\right]$

Multi-antenna channel $\mathrm{h}_{\mathrm{nm}}$ corresponding to the channel between the $\mathrm{n}^{\text {th }}$ transmission antenna and $\mathrm{m}^{\text {th }}$ reception antenna. (Fig. 1)

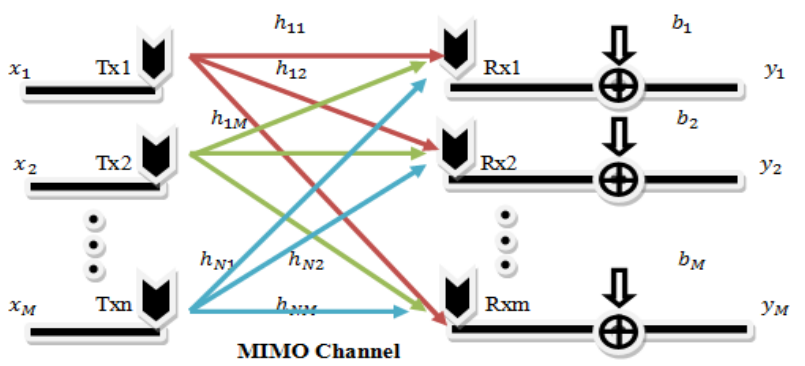

Fig.1. MIMO system $\mathrm{N} \times \mathrm{M}$

We consider the noise vector $b$ of the $M$ receives antennas such that:

$\mathrm{b}=\left[\mathrm{b}_{1} \ldots \mathrm{b}_{\mathrm{t}} \ldots \mathrm{b}_{\mathrm{N}}\right]^{\mathrm{T}}$

Then the received vector $y$ is expressed as follows:

$\mathrm{y}=\mathrm{Hx}+\mathrm{b}$

It is generally assumed that the noise is negligible able to reconstruct the transmitted signal. The expression (4) can be written:

$\mathrm{y}=\mathrm{Hx}$

Knowing $\mathbf{y}$ and $\mathbf{H}$, the relationship which allows reconstruction $\mathrm{x}$ is:

$\mathrm{x}=\mathrm{H}^{-1} \mathrm{y}$

Mathematically, solving this equation requires that $\mathrm{H}$ is invertible which means that $\mathrm{H}$ is full rank (its size is equal to its rank). Physically, this results in attenuation and different phase shifts suffered by each transmitted signal. This is why it is essential to have an environment rich in multipath to take full advantage of the contributions of a MIMO system.

\section{Capacity of SISO, MISO, SIMO and MIMO Channel}
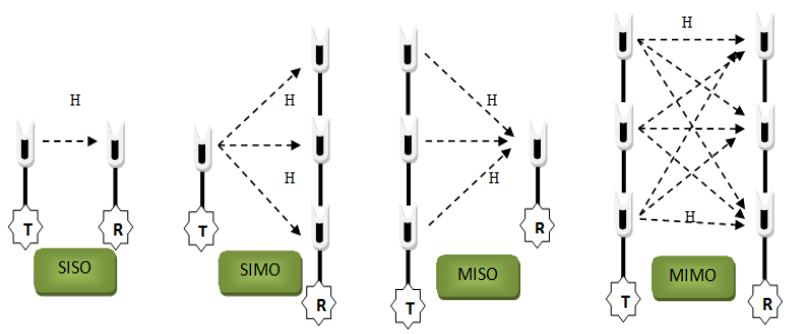

Fig.2. SISO, SIMO, MISO, MIMO Channels

In this section we will explain the expression of all wireless communication systems Fig.2 and we will concentrate to the capacity of MIMO channels Fig.1 and Fig.2. As we know Capacity is a performance measure for digital communication systems. It is the maximal transmission rate for which a reliable communication can be achieved [8]. If the transmission rate gets larger than the capacity, the system breaks down and the receiver makes decoding errors with a non negligible probability. Capacity is the primary tool to characterize the performance of MIMO systems and it also serves in practical system as a guide to properly design the transmitted signals as well as the processing of the received signals [8].

With the same way we will illustrate a comparison between the different multi-antenna systems; the purpose of this section is to evaluate how changing the transmission capacity in terms of antenna number. Here we will compare the capacity of various existing channels (SISO, SIMO, MISO and MIMO) without knowledge of the state of the CSI channel. The notion of channel Capacity was introduced by Claude Shannon by an expression (7) often known as Shannon's formula [9]-[11]:

$\mathrm{C}=\mathrm{W} \log _{2}(1+\mathrm{P} / \mathrm{N})$ bits/second

An expression (logarithmic function) for how many bits of information can be transmitted without error per second over a channel with a bandwidth of $W(\mathrm{~Hz})$ and signal to noise ratio $(P / N)$.

\section{A. Capacity of SISO Channel}

SISO system with the channel gain $h$, SNR the signal to noise ratio at the receiving antenna, capacity without knowing the CSI:

$\mathrm{C}=\log _{2}\left(1+\mathrm{SNR}|\mathrm{h}|^{2}\right) \quad \mathrm{Bit} / \mathrm{s} / \mathrm{Hz}$

Thus the theoretical capacity will be:

$$
\begin{gathered}
\mathbf{C}_{\text {Theo }}=\mathrm{E}(\mathrm{c})=\log _{2}\left(1+\operatorname{SNR} \cdot \mathrm{E}\left(|\mathrm{h}|^{2}\right)\right) \\
\text { Or } \mathrm{E}\left(|\mathrm{h}|^{2}\right)=1
\end{gathered}
$$

Thus: $\quad \mathbf{C}_{\text {Theo }}=\log _{2}(1+$ SNR $)$

[6]

The capacity increases as logarithmic function of (1+SNR).

\section{B. Capacity of SIMO Channel}

A channel SIMO (Single Input, Multiple Output) is a multiantenna system with a transmit antenna and $\mathrm{M}$ antennas at the reception. With $h_{i}$ the complex gain between the transmit antenna and the $i^{\text {th }}$ receiving antenna, its capacity will be:

$\mathrm{C}=\log _{2}\left(1+\operatorname{SNR} \cdot \sum_{\mathrm{i}=1}^{\mathrm{M}}\left|\mathrm{h}_{\mathrm{i}}\right|^{2}\right)$

Avec $\sum_{i=1}^{M}\left|h_{i}\right|^{2}=M^{2}$, its Shannon capacity is given by:

$\mathrm{C}_{\text {theo }}=\mathrm{E}(\mathrm{C})=\log _{2}\left(1+\mathrm{SNR} \cdot \mathrm{M}^{2}\right)$

We find that its capacity increases with the $\log$ of $\left(1+\mathrm{SNR} \mathrm{M}^{2}\right)$, faster than in the SISO case.

\section{Capacity of MIMO Channel}

With MIMO system multiple antennas are employed both at the transmitter and the receiver, the addition of multiple antennas at the transmitter combined with advanced signal 
processing algorithms at the transmitter and at the receiver gives a performance in terms of capacity and diversity[10]. A MIMO channel is a wireless link between $\mathrm{N}$ transmits and $\mathrm{M}$ receive antennas. It consists of $\mathrm{NM}$ elements that represent the MIMO channel coefficients. The multiple transmit and receive antennas could belong to a single user modem or it could be distributed among different users [10]. For a MIMO channel with $\mathrm{N}$ transmit antennas and $\mathrm{M}$ receive antennas, as we know $\mathrm{H}$ is the channel matrix of size $\mathrm{N} \times \mathrm{M}$.

$\mathrm{H}=\left[\begin{array}{ccc}\mathrm{h}_{11} & \cdots & \mathrm{h}_{1 \mathrm{~N}} \\ \vdots & \ddots & \vdots \\ \mathrm{h}_{\mathrm{M} 1} & \cdots & \mathrm{h}_{\mathrm{MN}}\end{array}\right]$

$\mathrm{h}_{\mathrm{ij}}$ is the complex channel gain between the $\mathrm{j}^{\text {th }}$ transmit antenna and the $\mathrm{i}^{\text {th }}$ receiving antenna:

$\mathrm{C}=\log _{2}\left(\operatorname{det}\left[\mathrm{I}_{\mathrm{M}}+\frac{\mathrm{SNR}}{\mathrm{N}} \mathrm{HH}^{\mathrm{H}}\right]\right)$

In this equation, "det" means determinant, $I_{M}$ means $\mathrm{N}$ $\times \mathrm{M}$ identity matrix and $H^{H}$ means Matrix transpose conjugate Where the numbers of antennas $\mathrm{N}$ and $\mathrm{M}$ are important, the Expected value function of capacity for a Rayleigh channel grows proportionally to $\mathrm{M}$.

$\mathrm{E}[\mathrm{C}] \approx \mathrm{M} \cdot \log _{2}(1+\mathrm{SNR}) \quad \mathrm{Bit} / \mathrm{s} / \mathrm{Hz}$

In conclusion, Capacity increases much faster in MIMO systems than in the SISO and SIMO thanks to the large number of antennas case.

\section{Comparison of Capacity for SISO, MISO, SIMO and MIMO}

Fig.3 shows the capacity of SISO and MIMO systems in terms of SNR for several values of $\mathrm{N}$ and M. [15]. In the SISO case $(N=1$ and $M=1)$ capacity ranges from 1 to 17 bps / Hz. It remains low and increases slowly with the SNR, which illustrates the limitations of SISO transmissions. Despite the current techniques to make the most of a SISO channel capacity remains limited and a multi-antenna system, even under-exploited, get better performance. With MIMO examples ( $\mathrm{N}=4$ and $\mathrm{M}=4)$ capacity ranges from 3 to $48 \mathrm{bps} / \mathrm{Hz}$ (3 times larger than SISO). It increases rapidly with the SNR, which illustrates the performance of a MIMO communication.

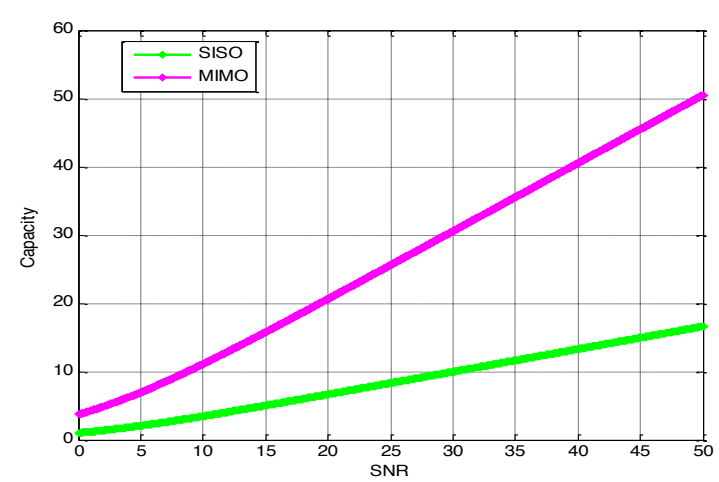

Fig. 3. Comparison in Capacity between SISO and MIMO.

To illustrate the increase of capacity according to the number of antennas for all systems we give the following (Fig.4) [8]:

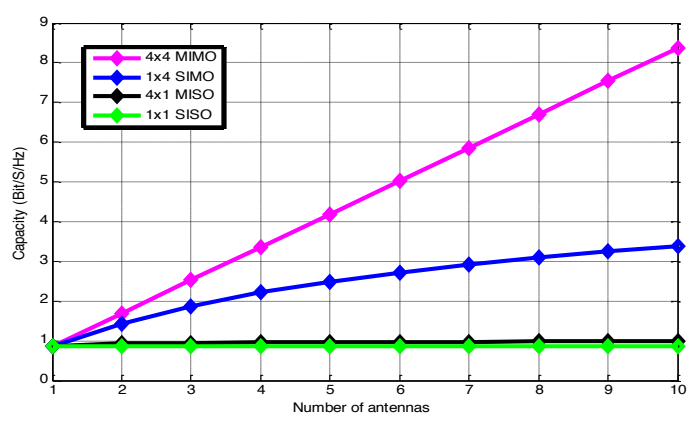

Fig. 4. Capacity vs number of antennas for MIMO, SIMO, MISO and SISO systems.

When we look at Figs 3 and 4, we observe that MIMO capacity grows linearly with respect to the number of antennas and it's approximately $\mathrm{M}$ times larger than SISO capacity ( $\mathrm{M}$ number of antennas). For MISO, the performance gain is negligible when the number of transmit antennas increases. For SIMO system it's more efficient than SISO and MISO system which ranges from 1 to $3.4 \mathrm{bps} /$ $\mathrm{Hz}$. and other remains connected to the value $1 \mathrm{bps} / \mathrm{Hz}$; it remains low with increasing number of antennas, which illustrates the limitations of SISO MISO transmissions. We can compare the variations of capacity of SIMO and MIMO systems based on the number of antennas for an SNR of $15 \mathrm{~dB}$ (for example) .Improved capacity is more pronounced for high SNR. For a SIMO system, the capacity follows a logarithmic trend when we increment the number of antennas at the reception while the MIMO system increases linearly when we increase the number of transmit and receive antennas. The advantage (in term of capacity) of MIMO systems is mainly due to the exploitation of multipath. First, they allow the receiver to distinguish the different transmitting antennas, and therefore to simultaneously transmit multiple symbols. Then, each path is a replica of the transmitted signal, and therefore carries useful information. We can also say that each path is equivalent to the direct signal emitted by a virtual antenna, which virtually increases the number of transmit antennas. The price to pay for this increase in capacity is first material, with the proliferation of antennas and their associated electronics, but also software with much more complex receivers and asking for more power calculation and therefore increased consumption. Finally, degradation of the correlation coefficient between the antennas can result in reduced capacity. By increasing the number of antennas for many MIMO systems capacity increases with, and we have the following (Fig. 5):

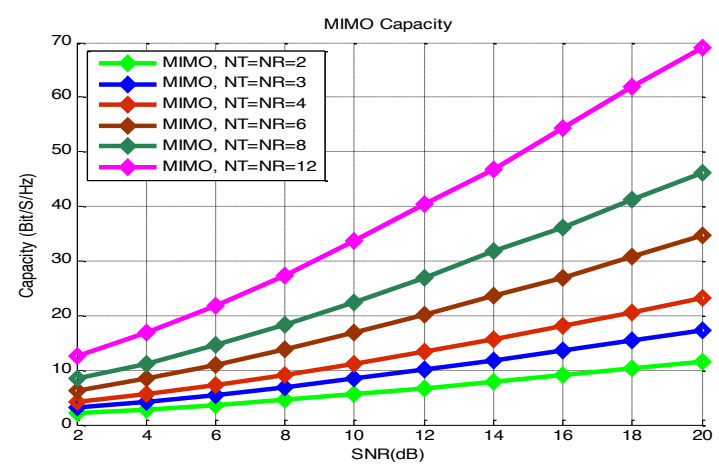

Fig.5. Capacity vs number OF MIMO antennas. 
When we compare these three pairs of antennas $\left(N_{T}=M_{R}=2\right),\left(N_{T}=M_{R}=4\right)$ and $\left(N_{T}=M_{R}=3\right),\left(N_{T}=M_{R}=6\right)$ and $\left(N_{T}=M_{R}=4\right),\left(N_{T}=M_{R}=8\right)$ we find that MIMO capacity is doubled 2 times; MIMO capacity then increases much more rapidly with the SNR, to finish with a gain of more than $50 \%$ at $20 \mathrm{~dB}$ SNR. After that when we look at the pair of antennas $\left(N_{T}=M_{R}=6\right),\left(N_{T}=M_{R}=12\right) \quad$ MIMO capacity then increases much more rapidly with the SNR, to finish with a gain of more than $70 \%$ at $20 \mathrm{~dB}$ SNR.

\section{Conclusion}

In this research, we have attempted to provide a clear image of the effect of using multiple antennas on the Capacity of wireless communication systems by using Matlab codes. Results generated show the relationship between Number of Antenna and Capacity; our results show that increasing the number of transmitting and receiving antennas for a wireless MIMO channel does indeed improve the channel capacity and performance that can be obtained.

Future wireless communication systems will perform a breakthrough in system performance, by taking use of antenna arrays at both sides of the communication link.

\section{References}

1. Lekun Lin "Choosing between open- and closed-loop MIMO in BTS systems" Embedded Computing Design — May 15, 2009

2. David Gesbert, Member, IEEE, Mansoor Shafi, Fellow, IEEE, Dashan Shiu, Member, IEEE, Peter J. Smith, Member, IEEE, and Ayman Naguib, Senior Member, IEEE “ From Theory to Practice: An Overview of MIMO Space-Time Coded Wireless Systems" IEEE JOURNAL on selected areas in communications, vol. 21, NO. 3, April 2003.

3. M. A. Jensen and J. W. Wallace, "A review of antennas and propagation for MIMO wireless systems, "IEEE Trans. Antennas Propagat., vol. 52, no. 11, pp. 2810-2824, Nov. 2004.

4. M. Bialkowski, "Research into multiple element antennas to enhance performance of wireless communication systems," in Proc. XVI Intrnl.Conf. on Microwaves, Radar, Wireless Communications, vol. 3, May 2006, pp. 1071-1082.

5. IAN POOLE, Radio-Electronics: Resources and Analysis for Electronics Engineers: What is MIMO? Multiple Input Multiple Out Put Tutorial.

6. E. Telater, "Capacity of Multi-Antenna Gaussian Channel", AT\&T Bell Labs, Tech. Memo., June 1995.

7. E. Telatar, "Capacity of Multi-Antenna Gaussian Channels", invited paper, European Transactions on Telecommunications, Vol. ETT-10, No. 6, pp. 585-595, 1999.

8. Tim Brown, Elisabeth De Carvalho, Persefoni Kyritsi "Practical Guide to the MIMO Radio Channel with Matlab Examples" this edition first published 2012.

9. C. E. Shannon, “A Mathematical Theory of Communication”, Bell Syst. Techn. J., Vol. 27, pp. 379-423, 623-656, July, October, 1948.

10. Nirmalendu Bikas Sinha, R. Bera, M. Mitra "Capacity and VBLAST techniques for MIMO Wireless" Journal of Theoretical and Applied Information Technology 2005 - 2010 JATIT.

11. G. J. Foschini and M. J. Gans, "On limits of wireless communications in a fading environment when using multiple antennas," Wireless Pers. Comm., vol. 6, no. 3, pp. 311-335, Mar. 1998.

12. 'I. E. Telatar, "Capacity of Multi-Antenna Gaussian Channels," Eur. Trans. Telecomm., vol. 10, pp. 2172-2178, 2000.

13. Vasanthan Raghavan and Akbar M. Sayeed "Multi-Antenna Capacity of Sparse Multipath Channels" Information Theory Workshop 2004 (ITW 2004), San Antonio, TX and the IEEE International Symposium on Information Theory 2006 (ISIT 2006), Seattle, WA.

14. Rafik Ahmad, Devesh Pratap Singh, Mitali Singh "Ergodic Capacity of MIMO Channel in Multipath Fading Environment" I.J. Information Engineering and Electronic Business, 2013, 3, 41-48 Published Online September 2013 in MECS.

15. Akhilesh Kumar, Anil Chaudhary "Channel Capacity Enhancement of Wireless Communication using Mimo Technology" International Journal of Scientific \& Technology Research Volume 1, Issue 2, March 2012. 